$\begin{array}{ll}\text { le portiQue } & \text { Le Portique } \\ \text { Revue de philosophie et de sciences humaines }\end{array}$

$20 \mid 2007$

Gilles Deleuze et Félix Guattari : Territoires et devenirs

\title{
Faire rhizome en passant en revue
}

\section{Anne Querrien}

\section{OpenEdition}

\section{Journals}

\section{Édition électronique}

URL : http://journals.openedition.org/leportique/1358

DOI : $10.4000 /$ leportique. 1358

ISSN : $1777-5280$

\section{Éditeur}

Association "Les Amis du Portique"

Édition imprimée

Date de publication : 15 décembre 2007

ISSN : 1283-8594

\section{Référence électronique}

Anne Querrien, «Faire rhizome en passant en revue », Le Portique [En ligne], 20 | 2007, mis en ligne le 06 novembre 2009, consulté le 25 mars 2021. URL : http://journals.openedition.org/leportique/1358 ; DOI : https://doi.org/10.4000/leportique.1358

Ce document a été généré automatiquement le 25 mars 2021.

Tous droits réservés 


\title{
Faire rhizome en passant en revue
}

\author{
Anne Querrien
}

1 Quand Liane Mozère m'a demandé de traiter du fonctionnement rhizomatique des Annales de la Recherche Urbaine j'ai légèrement déplacé l'angle d'attaque en parlant de "faire rhizome», comme d'autres font société. Faire société c'est réunir la communauté autour d'un vide investi des pouvoirs transcendantaux qui autorisent le partage égalitaire de la parole ou des richesses, mais aussi normalisent la subjectivité en un appendice de la défense d'un bien commun qui se résumerait à cette égalité ; faire société c'est méduser la subjectivité en miroir de son devoir être. Le mouvement des banlieues nous montre bien que la subjectivité déborde de ces bienséances de partout. Quand j'ai commencé à travailler avec Félix Guattari en 1965 ce débordement était plus limité, moins perceptible en tout cas pour moi, sauf déjà dans ces trajectoires très minoritaires qui aboutissaient au lieu de soins psychiatriques. La folie s'exprime précisément dans ce qui pour les non fous est une affirmation d'une incapacité de vivre ensemble. Comment alors agir une ou d'autres formes de vivre ensemble, agir l'impératif démocratique, si la forme normale est impraticable ? En faisant rhizome, en faisant lien autrement, en partageant non pas autour d'un cercle, mais de proche en proche, en construisant l'égalité au sein des différences de potentiel par la transmission. Ce n'est pas un autre projet de société que nous avons commencé de mettre en place, mais d'autres manières d'être dans la société, horizontales, non hiérarchiques: faire des passes plutôt que marquer, ouvrir le jeu plutôt que se faire remarquer.

2 J'ai rencontré l'expression «Faire rhizome » dans la bouche de Félix quelques années avant qu'elle soit éditée, à propos d'un journal que j'avais tenu en son absence, sur le quotidien du CERFI (le centre de recherches en sciences sociales où nous travaillions ensemble), et qui relatait mes errements par rapport à ce groupe qui me repoussait autant qu'il m'attirait. Écriture d'une série de textes scandée par le renouvellement des jours, avec la maniaque attention de couvrir chaque jour, et mise en évidence de ce mouvement de stop and go, de fuite et de décision, de gestion d'un site existentiel traversé d'affects contradictoires. Par rapport à un attachement, ou plutôt à des 
attachements vécus comme ligotant, l'écriture dessinait une tangente, la trajectoire au jour le jour d'un point qui restait là, au milieu.

3 De ce moment, j'ai retenu l'idée que le rhizome avait à voir avec la visagéité, les trous noirs et le mur blanc, qu'on trouve dans un autre passage de Mille Plateaux. Faire rhizome permet d'échapper à la contemplation du visage du despote et à la nécessaire soumission, même réactive qui s'ensuit. Faire rhizome c'est choisir de s'écarter même infinitésimalement du chemin tracé qui conduit droit au trou noir, à la maximisation de l'affect ; c'est une micropolitique du désir, une conduite même lente du déplacement vers le dehors vers lequel tend aussi le désir, une lutte parfois difficile contre l'effondrement au centre duquel attire la passion pour le semblable.

Faire rhizome c'est :

- prendre les choses par le milieu

- parcourir l'espace de proche en proche et le faire proliférer

- développer une forme de connaissance exploratoire, indicative, anexacte

- développer une forme littéraire mineure

- mettre sur pied un dispositif machinique de production répétitive

- produire une série de raison indiscernable

5 Cette notion de rhizome va être développée à partir de l'expérience de la revue Les Annales de la Recherche Urbaine mais de nombreuses productions intellectuelles ou artistiques prennent cette forme rhizomatique. Je pense en particulier à la série de visages de femmes produite par la graveuse Sonja Hopf.

\section{Prendre les choses par le milieu}

6 Je ne sais pas très bien ce qu'est un rhizome pour de vrai, mais des explications que m'en a données Félix j'ai retenu l'idée que c'était une plante qui pouvait se repiquer et refaire des racines, comme le riz, ou pour moi le géranium, fleur très mal vue des intellectuels mais qui j'ai bien pratiquée en vacances. Pas de racines, on peut reprendre une revue qui existe, au milieu de tout, parce que son fondateur, Jean-Claude Daumas, meurt, dans le cas des Annales de la Recherche Urbaine, ou pour n'importe quelle raison, et notamment du fait du pouvoir despotique des financeurs, pour beaucoup de revues qui m'entourent. Une revue cela a la vie dure, ce n'est pas liée à une personne, contrairement aux apparences de l'instant, c'est une série.

7 La revue est au milieu de plein d'autres revues, elle baigne dans un milieu de revues, qui la caractérisent par ricochet, lui donnent ses proximités, ses spécificités, balisent son espace.

8 Au jour le jour la revue apparaît isolée, surtout si elle représente ses auteurs et ses lecteurs, car ces deux milieux sont en perpétuel changement, en glissement, et c'est précisément ce glissement qu'elle est chargée d'explorer, dont elle doit rendre compte, qu'elle doit évaluer.

9 Nous sommes plusieurs revues à essayer de rendre compte du milieu urbain en France chacune à sa manière, avec son propre dispositif de rédaction, avec ses propres moyens, sans arriver à couvrir tout ce que nous sentons de cette réalité en perpétuelle transformation. Je pense notamment outre les Annales de la Recherche, Urbaine à Urbanisme, Espace et Société, Espaces-Temps, Territoires, Traits urbains. Alors chacun propose son approche, avec le même sentiment d'incomplétude. Chose étrange, ces 
visions différentes paraissent plus complémentaires, voire convergentes, que concurrentes, comme si le fait de chausser des lunettes institutionnelles pour regarder la réalité sociale en transformation y découpait les mêmes images, y laissait dans l'ombre les mêmes pans bien plus vastes.

10 Le milieu des revues ne se limite pas à l'urbanisme ; toutes les revues sont entre revues, comme le souligne par son titre Ent'revues le salon annuel des revues culturelles. Un réseau européen de soixante-dix revues littéraires et culturelles vient élargir le paysage avec le magazine et le site Eurozine.com. Dans cet univers en réseau je me distingue cependant par l'appartenance à plusieurs rédactions de revue : Multitudes et Chimères à côté des Annales de la Recherche Urbaine, des revues plus proches politiquement et analytiquement du travail de Félix Guattari et Gilles Deleuze. D'habitude on représente une revue, on se sert d'une revue pour s'exprimer, on ne vit pas entre plusieurs revues, dont on choisit l'une ou l'autre suivant les facettes de ce qu'on est en train de faire. Les revues sont disciplinaires, liées à une méthode ou à un objet universitaires. Elles valorisent le travail de recherche effectué dans les laboratoires, elles le soumettent à la discussion de la communauté scientifique dont les deux referees qui lisent le projet d'article au nom de comité de rédaction sont l'avant-garde éclairée, choisis d'ailleurs pour être les plus proches du point de vue de l'auteur, et donc les plus à même pour le juger et pour le tirer dans le droit chemin.

11 Aux Annales de la Recherche Urbaine le travail est envisagé différemment: les articles, écrits en réponse à des appels à contribution pour des numéros thématiques, sont considérés par nous comme des prélèvements sur le milieu de la recherche urbaine, dont la composition au sein du numéro en préparation, va donner une image de la problématisation du sujet, pour ce que nous en connaissons (et nous ne connaissons pas toujours tout). Les recherches auxquelles il est fait appel sont des recherches en cours, et les articles rendent compte plus de problématisations que de résultats. Leur juxtaposition dans le numéro essaie de donner la vision de comment le milieu de la recherche urbaine envisage de penser telle ou telle question. Si suite à notre appel à articles, il manque un ou plusieurs pans problématiques que nous avons déjà repérés, nous chercherons de nouveaux articles quitte à outrepasser les décisions du comité de rédaction, et à devoir engager une procédure de réparation à son égard.

La fuite des numéros chemin faisant est d'ailleurs quasi inévitable, car au fur et à mesure qu'on travaille à la mise en forme des articles, et qu'on découvre en notes de nouvelles références, le paysage du problème étudié se transforme et donne envie d'autres contributions. Or nous sommes contraints par un budget et un nombre de pages et cette ouverture à l'infini n'est pas possible.

On considère généralement dans les revues que le rédacteur en chef est un super lecteur, premier lecteur, et que son jugement doit donc porter sur la clarté de la rédaction. C'est aussi un élément de notre position, mais auquel s'ajoute le travail de composition du numéro et le soin de relier entre eux des articles arrivés à l'issue d'un même appel à articles, mais de manière séparée, inclassable. Les propositions de corrections des textes incorporent de petites suggestions qui visent à coudre l'ensemble, ou à éviter les redondances d'un article à l'autre. L'appariement des articles est aussi un élément important: essayer sur un même angle d'attaque du problème d'avoir plutôt deux articles qu'un, dédoubler les perspectives. Le devenir-lecteur que dessert le travail du rédacteur en chef, se diffracte dans une multiplicité de sensibilités 
possibles au milieu desquels doit se situer le numéro, traitant chaque point de vue également, tout en respectant le point de fuite des hypothèses de départ.

Un numéro n'est en effet lancé que si nous avons une hypothèse sur le sujet que nous mettons en rédaction, si nous arrivons à nous situer au milieu du milieu, comme un agent de dynamisation, de composition, d'interrogation. Ce sujet il ne vient pas du milieu des chercheurs auquel nous avons accès de manière beaucoup trop partielle, mais du milieu des professionnels et des politiques qui se débattent avec des questions qu'ils n'arrivent pas à résoudre, et par rapport auxquelles ils sont prêts à revoir leurs représentations. Actuellement par exemple il y a une forte demande de recherche économique chez les professionnels et les politiques d'une part pour comprendre la croissance des coûts de l'immobilier et du foncier, nettement plus rapide que celle de l'économie dans son ensemble, d'autre part pour savoir équilibrer les opérations d'aménagement là encore en termes et coûts et d'investissements. Il nous semble qu'une telle problématisation loupe des questions abordées dans le cadre du travail avec d'autres revues, (Multitudes notamment), sur la mutation de l'économie en direction du travail cognitif ou immatériel et sur la remise en question de la loi de la valeur que cela induit. Cela semble aussi très loin des affirmations de la Commission européenne, selon lesquelles il faut développer une économie de la connaissance la plus productive du monde. Comment mettre en relation les différents éléments de notre propre réseau de connaissances et poser la question de l'économie de la connaissance à l'échelle d'une ville ou d'un territoire régional? Il n'y a pas de lieu constitué pour penser ces questions, mais des bribes abordées ici et là. Un numéro de revue ne va constituer qu'un élément de visibilité de la question, très temporaire, et très limité, mais suffisant pour que la question rebondisse. Cela peut sembler curieux de faire ainsi une revue au milieu des recherches et en amont des résultats, alors qu'on attendrait de cette revue qu'elle publie des résultats confirmés. Cette habitude s'est prise de façon pragmatique: une fois la recherche achevée le chercheur passe à autre chose, et l'écriture n'est plus mue par le désir de trouver; elle devient plus lourde, elle démontre et elle ennuie.

\section{Arpenter le milieu de proche en proche et le faire proliférer}

Le milieu de la recherche urbaine, aux confins des disciplines des sciences sociales, et en articulation aux interrogations des politiques et des professionnels est en mouvement constant. Il est traversé par des lignées fondatrices le long desquelles se renouvellent les sujets abordés par des méthodes semblables, mais aussi perpétuellement troublé par de nouveaux questionnements qui le rassemblent provisoirement sur des nouvelles questions. La revue joue un rôle de médiation, de transmission, de mise en proximité des questions des professionnels et des chercheurs, ou de mise en visibilité de recherches relativement isolées, mais considérées novatrices parce qu'elles s'approchent davantage des nouvelles questions des professionnels. C'est ainsi que des sociologues ou des géographes, principalement, ayant eu affaire à un problème en tant que citadins, vont devenir chercheurs urbains pour tenter de le comprendre de l'intérieur. C'est ainsi aussi que des sociologues ou des géographes plus théoriciens, peuvent être appelés à participer à la rédaction de la revue, parce que leur problématique semble présenter des parentés avec les problématiques urbaines. 

de commissions de recrutement tant pour les Universités que pour le CNRS apparentant la recherche urbaine, ou plutôt l'urbanisme, à la géographie, ne suffit pas à délimiter les contours d'un souci de l'urbain qui puise à toutes les disciplines. La revue ne fournit pas non plus un espace de publication suffisant pour donner à la face urbaine de toutes les disciplines un espace régulier d'expression, qui donnerait une représentation relativement exhaustive des recherches en cours. Ces recherches menées souvent dans le cadre de thèses ou de contrats de recherches, ne font pas l'objet d'un travail de synthèse et d'élaboration de résultats, mais restent relativement dispersées, et la revue ne peut que prélever sur cet ensemble ce qui se rapproche du thème qu'elle développe à ce moment-là. motivées par le même ensemble de questionnements. Pourtant on constate qu'elles restent juxtaposées dans leurs formulations finales malgré les efforts faits par les gestionnaires de recherches pour faire dialoguer les chercheurs. On arrive davantage à baliser les différents constituants d'une thématique qu'à formuler une alternative nette à la pensée dominante, ou des préconisations d'action. La temporalité des appels d'offres s'étale sur environ quatre ans entre le moment où on se saisit de la question, celui où on la transforme en questionnement, le jury et la passation des contrats, le rendu des rapports de recherche environ deux ans plus tard. Les questions se sont déplacées entre temps et de nouveaux chercheurs non invités à travailler après l'appel d'offres sont apparus, ont travaillé entre temps et contribuent à constituer le champ problématique considéré.

18 Il y a donc un décalage entre l'exploration d'une problématique par la revue, le défrichement du milieu dans toutes les directions possibles, et le calage d'un appel d'offres. Les publications collectives d'articles réalisées après l'appel d'offres, se limitent aux recherches reconnues par le jury d'appel d'offres, et ne se proposent plus d'explorer le milieu pertinent, de l'étendre et notamment de suivre l'émergence toujours renouvelée de jeunes chercheurs.

19 Un milieu vivant est un milieu sans fin et non un milieu délimité par un jugement, que ce jugement émane d'un jury ou des commissions du CNRS. C'est du mouvement de la recherche ce faisant dont il s'agit de rendre compte, et non du seul stock de chercheurs reconnus. Telle est la conviction qui anime la revue et qui fait courir toutes les occasions où de jeunes chercheurs (des chercheurs jeunes dans la recherche, quel que soit leur âge à l'état civil) peuvent se manifester, en particulier les colloques et les bulletins de laboratoire. Il faudrait pouvoir se démultiplier pour y arriver.

Le travail de la rédaction au sein de ce milieu en expansion, et qu'il s'agit de faire proliférer en introduisant des liens non encore pratiqués est un travail d'agencement, de composition, non pas l'expression d'un école de pensée, mais la mise en relation de plusieurs écoles dans une atteinte à un problème et derrière à cette question de la connaissance de la ville et de sa gestion. Toutes les recherches sont bonnes à prendre du moment qu'elles se proposent comme une manière de traiter le thème proposé pour le numéro en cours, et qu'elle se proposent comme une manière non définitive, une interrogation ouverte. Il n'y a pas de débat explicite dans la revue qui retranscrirait un débat que nous aurions mené oralement auparavant, et la composition d'articles ne forme pas vraiment débat, mais kaléidoscope, renvoyant le lecteur à des facettes dont il faudra poursuivre l'approfondissement ailleurs. 
21 La revue est un moment dans un développement, un moment que nous essayons de situer plutôt en début du processus de pensée sur le thème considéré, et nullement en position de colloque final ou de déclaration de synthèse. De ce point de vue la revue est plus proche des magazines d'information professionnelle que des revues scientifiques proprement dites, quoiqu'on s'aperçoive que dans le champ de la recherche urbaine, les revues dites scientifiques ont tendance à se comporter de la même manière. Il semble que ce soit en cartographiant le champ urbain qu'on le fasse exister tant comme problème que comme pratique sociale, comme mouvement de transformation de la société par elle-même. Les travaux des précurseurs dans le champ, de l'École de Chicago à Henri Lefebvre ont tous pris ce tour.

\section{Une forme de connaissance exploratoire}

22 Vingt ans d'animation de la revue les Annales de la Recherche Urbaine ne me donnent pas le sentiment de connaître la ville, mais plutôt un grand nombre de façons de l'aborder toutes partielles et qui peuvent répondre à des questions de professionnels, d'élus ou de citadins très partielles également. Nous avons des bouts d'explication, mais nous n'avons aucun instrument sérieux de maîtrise des phénomènes comme la ségrégation, la pauvreté, les inégalités. L'analyse globale grâce aux instruments statistiques et cartographiques ne peut donner lieu qu'à des mesures partielles, dont les effets ne sont absolument pas garantis. Ce que peut faire la revue, et qui la rapproche des multiples formes de publication contemporaines, aussi bien de la Commission européenne, que de multiples réseaux d'activistes plus ou moins virulents, c'est informer sur les actions de changement et leurs effets apparents, quels que soient les acteurs qui les entreprennent, informer sur la ville comme un grand champ de recherche, d'efforts de connaissance et de transformation.

23 La revue informe sur la ville comme un espace de changement, parcouru de nombreuses lignes de transformation, mais généralement non sécantes, non situées dans les mêmes plans. Petit à petit la conviction démocratique selon laquelle c'est l'habitant, l'usager qui va condenser ces forces de changement de façon positive s'est effacée, sans pour autant être remplacée par la confiance dans l'action des professionnels qui non seulement reste sectorielle, mais exclut généralement une partie de la population, sauf quand elle est spécifiquement dédiée à s'occuper d'elle. Le libéralisme se marque dans les articles que nous recueillons, et même dans notre questionnement, où la capacité d'initiative est implicitement une capacité financière. La ville d'aujourd'hui est faite par ceux qui en ont les moyens, et la réflexion sur la ville se fait à partir de leurs choix, de leurs problèmes, y compris à partir de leur compassion et de leur refus de l'indifférence.

24 Dans les années 1980, le moteur de l'espace exploré par la revue était constitué par des recherches qui accompagnaient des expériences de recherches-actions très liées aux habitants et à la production d'un espace de vie plus approprié à leur quotidienneté que celui qui était formaté aux normes nationales. Mais cet espace pensé et transformé avec les habitants, n'a pas été accompagné d'une gestion renouvelée des attributions, ni des transformations économiques et sociales nécessaires à la poursuite dans la durée des transformations initiées. La valeur d'interrogation de ces recherches-actions par rapport à la production urbaine normale a disparu. Les nouveaux espaces de transport cependant ont permis de donner à la recherche un champ d'expérience, comme par 
exemple la construction de la ligne Meteor de la RATP, suivie par Isaac Joseph, ou les constructions de nouvelles gares pour accueillir le TGV.

Un autre moteur de recherche plus critique est apparu avec l'emprise croissante dans la pratique de néologismes issus de travaux internationaux, en général en langue anglaise : développement durable, cohésion sociale, gouvernance, partenariat publicprivé. La revue s'est efforcée d'explorer de quoi il s'agissait, quel champ de travail urbain cela pouvait dessiner, en quoi ces néologismes et les types d'expériences auxquelles il faisait référence à l'étranger pouvaient ouvrir de nouveaux champs de recherches, favoriser cette prolifération des situations d'exploration à laquelle nous nous sommes attachés. L'espace de travail pour la revue n'est plus orienté de la même manière dans ce cas. Il ne s'agit plus de faire remonter d'expériences au bas de l'échelle sociale, des propositions transversales indiquant de nouvelles possibilités. Il s'agit de partir d'injonctions qui se voudraient dominantes, via les décisions européennes, et de les latéraliser, de les mettre sur le côté, sans les ignorer, pour les apprivoiser et se donner les moyens de travailler les nouveaux problèmes qu'elles désignent. Ce n'est pas facile car l'effet de domination reste présent pour beaucoup de chercheurs. La matière recueillie ainsi a toutes les difficultés pour échapper à la langue de bois.

Pourtant le tropisme international nous aspire inexorablement. La méthode rhizomatique d'exploration, d'horizontalisation des relations, de mise en place de lignes de fuite à partir des effets miroir produits par les objets centraux, sera particulièrement utile pour résister à ce qui se présente comme une gigantesque entreprise de classement à l'échelle de la planète, une mise en hiérarchie servile dans l'obéissance aux critères unidimensionnels, qui laissera moins que jamais la place aux affects subjectifs qui déterminent le désir de chercher. Comment prendre cette aspiration dans l'autre sens? En gardant la distance sans ignorer, en incluant dans nos objets les injonctions et en les faisant proliférer, comme de nouvelles thématiques à prendre dans leurs ancrages concrets comme les autres, dans les expériences de base non pas qu'elles suscitent, mais auxquelles elles ressemblent, en coupant les relations de pouvoir pour comparer, relier, réagencer.

\section{Une forme littéraire mineure}

La revue est une forme littéraire mineure, déjà pour celui qui écrit un article qui n'a pas l'importance d'un livre, mais surtout pour celui ou celle qui fait écrire les autres et qui n'écrit pas lui ou elle-même, sauf peut-être une introduction. Cette introduction touchant à chaque numéro un sujet différent ne dessine pas une carrière, la naissance d'une autorité sur un sujet déterminé. Elle donne peut-être à penser par son questionnement, sur le moment, mais elle ne fait pas date. Et d'ailleurs on observe que ces introductions, voire les numéros de revues eux-mêmes pris comme ouvrages collectifs ne font l'objet que de très peu de citations. Le nombre de citations étant ce qui définit l'œuvre majeure l'affaire est entendue.

Mais s'agit-il de faire une œuvre majeure ? En fait notre recherche est très différente et entre dans les textes qui nous sont proposés. Nous sommes à la recherche de ce qui serait une tête chercheuse, de ce qui sortirait de la répétition des textes précédents du même auteur, ou de ce qui est avéré dans la communauté. Nous sommes à la recherche dans le texte d'un article de cet écart à la langue de la maîtrise qui signale la puissance de recherche à l'œuvre, la capacité de prolifération que nous cherchons à mettre en 
œuvre dans notre exploration du milieu. Qu'est-ce qui fait qu'un texte est différent, un tant soit peu, vise à rendre compte des différences dans l'expérience de tous les jours? Qu'est qui fait qu'un texte va être perçu comme différent, va être lu, par un lecteur professionnel non chercheur (la moitié des lecteurs de la revue), en même temps qu'il situera le chercheur dans son milieu de référence? Est-ce qu'un texte est lu en référence aux autres qui l'entourent comme nous le souhaiterions dans notre travail de composition? Ou est-ce qu'un texte est lu en référence à son auteur, auquel cas il faudrait n'en avoir que de très connus, au lieu d'explorer inlassablement un milieu qui se renouvelle par des inconnus forcément? Les nouvelles technologies sont ambiguës : elles donnent l'impression de trancher en faveur du majeur, la vente à l'unité, sur le nom de l'auteur principalement, d'articles garantis excellents par un comité de rédaction lui-même excellent. La forme même du défilement des pages sur l'écran isole l'article de son environnement matériel. En même temps les blogs donnent à chacun la possibilité de livrer au monde entier sa production et sa pensée la plus individualisée. Le travail d'un comité de rédaction, d'une revue comme espace intermédiaire entre l'internet et le papier, entre des lecteurs de niveaux très différents, entre les langues et les continents, doit sûrement être repensé.

Faut-il donner davantage à voir sous forme d'un article plus construit ce travail de composition, d'exploration, du milieu qui disparaît presque lorsque l'objet papier dont il était le résultat est abandonné ? Il semble, à travers l'expérience des documents de synthèse des appels d'offres du Plan urbanisme construction architecture, où est éditée la revue, que la fabrication d'un article à vocation majeure à partir de l'ensemble des contributions rassemblées, donne une représentation et une limite, brise l'effet rhizomatique possible d'une composition collective.

Dans la présentation collective les articles sont souvent courts, des éléments de présentation étant apportés par d'autres, la rédaction ayant enlevé les effets de redondance possible; les articles sont prélevés sur les documents initiaux. Dans une présentation isolée au contraire le contexte doit être inclus, et l'article vaut pour tous les autres, il est forcément le meilleur, garanti par un jury. La production de revue s'inclut dans la production de classement et de hiérarchie.

31 La revue est donc prise entre de nouvelles tensions liées au probable abandon des technologies traditionnelles de transmission par l'impression. La technologie internet semble emporter d'autres règles d'énonciation, d'autres formes d'agencement collectif qui visent à exhausser encore plus d'excellence individuelle, mais qui vont former nécessairement des restes, à partir desquels il sera sans doute possible de rhizomatiser en tension avec l'excellence, entre deux, dans de nouvelles conditions, encore à découvrir.

\section{Une machine abstraite}

Le dispositif de rédaction qui s'est mis peu à peu en place aux Annales de la Recherche Urbaine est devenu capable de traiter n'importe quel sujet du champ urbain avec un minimum de consistance malgré la dispersion de ces sujets. Par exemple les derniers numéros parlent de l'économie territoriale de la connaissance, du vieillissement de la population, des rapports entre intérêt général et intercommunalité, des villes nouvelles aujourd'hui, du renouvellement urbain, des liens religieux en ville, de la sensibilité aux risques, et ainsi de suite. Certes ces sujets appartiennent à un champ bien déterminé, 
mais aucun chercheur ne traiterait l'ensemble, il s'agit à chaque fois d'une composition différente.

Le dispositif comprend tout d'abord la détection du thème qui fait problème aux professionnels et qu'il conviendrait donc d'éclairer de diverses manières avec les chercheurs. Une hypothèse de départ plus ou moins intuitive est mise en place par l'équipe de rédaction pour introduire une mise en résistance du thème par rapport aux injonctions administratives ou professionnelles :

- l'économie de la connaissance ne se résume pas aux laboratoires de recherche connectés aux entreprises, elle englobe tous les cadres sociaux de la connaissance collective qui lient les acteurs locaux entre eux

- le vieillissement de la population c'est d'abord le surgissement d'énormément de temps libre et de capacité de participation citoyenne, et pas simplement le besoin de plus de maisons de retraite.

- l'intérêt général n'est plus énoncé par les fonctionnaires d'état mais par des compositions politiques locales, notamment intercommunales

- pendant qu'on fait le bilan des villes nouvelles françaises, les villes nouvelles se développent à vive allure dans le monde comme lieu d'habitat des nouvelles classes moyennes

- le renouvellement urbain qui consiste en France à rénover le logement social en y introduisant la " mixité sociale par le haut ", consiste ailleurs à rénover les espaces publics et à transformer les pratiques urbaines et pas seulement l'image sociale des quartiers défavorisés

34 Avec ce fil directeur, plus ou moins à contretemps du point de vue courant, une recherche documentaire est effectuée qui fournit, avec l'aide du comité de rédaction une liste d'auteurs potentiels. Un appel à articles est rédigé qui synthétise de manière interrogative toute cette matière. Les chercheurs ont deux mois pour répondre, afin que leur texte soit prélevé sur une recherche en cours, et non élaboré péniblement à partir de recherches passées. Les propositions d'articles sont sélectionnées par le comité de rédaction pour que le nombre ne dépasse pas nos possibilités budgétaires, alors qu'idéalement selon mon modèle proliférant et d'écriture mineure il faudrait tout garder. Là il y a un compromis entre le rhizome et la représentation. Le rhizome revient dans le travail sur les textes le long de la ligne de recherche de chacun. Les illustrations, quant elles ne sont pas données par les auteurs, ce qui est plus économique, sont choisies en agence, ce qui permet aussi d'introduire une tension supplémentaire. Quand le numéro est publié, on s'occupe un peu de sa diffusion, mais il vit sa vie, on passe à autre chose, c'est sur la rédaction qu'on investit.

\section{Les limites de l'opération}

Quel est le sens de cette accumulation de numéros, de ce balisage apparemment sans fin du champ de l'urbain? Chose curieuse, ce balisage n'épuise absolument pas la question, la ville reste un trou noir, une terra incognita, dont il surgit toujours des évènements qu'on peine à comprendre comme les émeutes de novembre dernier. Certes la technocratie a un besoin énorme de renouveler en permanence ses mots d'ordre pour servir à quelque chose, et dans la mesure où nous lovons notre travail tout contre ces mots d'ordre, pour les prendre à revers, nous avons du pain sur la planche. Mais il y a une très grande difficulté à établir le rhizome à travers tout le champ social, à établir des relations sociales à égalité, quand les distances sociales se font plus fortes. 


\section{RÉSUMÉS}

L'expérience de la revue Les Annales de la Recherche Urbaine est relatée à l'aide de quelques concepts de Deleuze et Guattari : faire rhizome en rencontrant les auteurs de proche en proche, en faisant proliférer le milieu de référence, en suivant ce qui le met en mouvement. La ville est explorée comme un espace aux entrées multiples, inconnaissable globalement. La revue résiste contre la mise en mots d'ordre du réel par l'agencement de ses contributions; elle construit la ville au jour le jour.

To produce a rhizome while editing a journal. The experiment of Les Annales de la Recherche Urbaine magazine is overviewed here with some concepts from Deleuze and Guattari: to produce a rhizome through the relations with the authors, to expand the milieu that they constitute, to follow its social movement. City is explored as a space with a multiplicity of ways in, with no possible global knowledge. The journal resists against reducing the world to key-words, by the agency of its contributions. It works to build the everyday city.

Rhizome ablegen, Revue passieren lassen. Die Autorin berichtet anhand einiger Konzepte von Deleuze und Guattari über das Experiment der Revue „Les Annales de la Recherche Urbaine"(Revue über städtische Forschung). Durch die Argentierung ihrer Abhandlungen übt 
diese Revue einen Widerstand aus: gegen die ordnungsbringende Ausdrucksweise der Realität erbaut die Stadt jeden Tag aufs Neue.

\section{AUTEUR}

\section{ANNE QUERRIEN}

Anne Querrien, sociologue-urbaniste, est responsable de la rédaction des Annales de la Recherche Urbaine au Plan urbain puis au Plan Urbanisme Construction Architecture, au Ministère de l'Équipement, depuis 1985. Elle a été accompagnée dans ce travail par Pierre Lassave, sociologue, de 1992 à 2006, puis par Marie-Flore Mattei, géographe depuis 2006. Elle participe également aux revues Multitudes et Chimères. Elle est l'auteur de « L'ensaignement » (Éditions, Recherches 1976), réédité aux Empêcheurs de penser en rond en 2005 sous le titre « L'école mutuelle ». Elle a participé à des nombreux ouvrages collectifs sur la ville et la recherche urbaine. 